\title{
Improvement of feed pellet characteristics by dietary pre-gelatinized starch and their subsequent effects on growth and physiology in tilapia
}

\begin{abstract}
A 9-week study was conducted to compare dietary corn starch (CS) or tapioca starch (TS), with or without being pre-gelatinized (PG), on the growth, feeding efficiencies, plasma and muscle biochemistry, intestinal short chain fatty acids (SCFA), and liver glycogen of triplicate groups of 20 red hybrid tilapia (Orecohromis sp.). Various pellet characteristics were evaluated, along with their surface and cross sectional microstructure. The PG diets had significantly higher water stability, bulk density, and protein solubility, along with a smoother surface. Tilapia fed the TS diet had lower growth than had all other tilapia, but were significantly improved when diet was pre-gelatinized. In the PG treatments, intestinal SCFA significantly decreased while plasma glucose, cholesterol and triglycerides, as well as liver glycogen, significantly increased. Fish fed the CS diet had significantly more long chain polyunsaturated fatty acid than had those fed by other treatments. Pre-gelatinization may improve fish productivity and offer greater flexibility during aquafeed production.
\end{abstract}

Keyword: Gelatinization; Short chain fatty acids; Glycogen; Pellet characteristics; Tapioca starch; Tilapia 